\title{
Review
}

\section{Methods for Evaluation of the Oxidative Stability of Lipid-Containing Foods}

\author{
Fereidoon SHAHIDI and Udaya N. WANASUNDARA \\ Department of Biochemistry, Memorial University of Newfoundland, St. John's, NF, Canada, AlB $3 X 9$
}

Received January 30, 1996

\begin{abstract}
Lipid oxidation and associated changes are major causes of quality deterioration of lipids and lipid-containing foods. Oxidative deterioration of lipids directly affects their flavor, color and nutritive value. In addition, free radicals formed during this process may also damage membranes, enzymes, vitamins and proteins that may be involved in the process of ageing. Thus, assessing the extent of oxidation in foods is necessary. This paper reviews the conventional methods as well as new procedures for determining oxidative stability of lipids. Criteria for selection of methods depend on a number of factors including the nature and history of sample, the type of information required, the time available for experimentation and test conditions.
\end{abstract}

Keywords: lipids, fats and oils, oxidative stability, methodologies

Autoxidation is a major deteriorative reaction affecting food lipids and is a primary concern for both the processing industries and consumers as it affects economics, nutrition, flavor, safety and storage of products (Labuza, 1971; Frankel, 1980). Other oxidative processes such as photooxidation and hydrolytic rancidity may also be encountered. Both of these latter processes may be controlled by cold storage, proper packaging or inactivation of enzymes. However, since autoxidation is a chemical reaction with a low activation energy (4$\left.14 \mathrm{kcal} \cdot \mathrm{mol}^{-1}\right)$, the rate of this reaction is not significantly diminished by lowering the temperature of food storage.

Extensive research has been carried out to elucidate the mechanism of autoxidation and to devise methods to control lipid oxidation in foods. Various oxidative products and their possible interaction with food components and biological molecules have been studied (Horton \& Fairhurst, 1987; Kanner et al., 1987). The oxidation of oils occurs at sites of unsaturation and proceeds via a free-radical chain mechanism (Labuza, 1971) consisting of initiation, propagation and termination steps.

Initiation starts with the abstraction of a hydrogen atom adjacent to a double bond in a fatty acid ( $\mathrm{RH})$ moiety and this may be catalyzed by light, heat or metal ions to form a free radical (Reaction 1). The resultant alkyl free radical $\left(\mathrm{R}^{*}\right)$ reacts with atmospheric oxygen (triplet state) to form an unstable peroxy free radical (Reaction 2) which may in turn abstract a hydrogen atom from another unsaturated fatty acid to form a hydroperoxide $(\mathrm{ROOH})$ and a new alkyl free radical (Reaction 3). The new alkyl free radical initiates further oxidation and contributes to the chain reaction. The chain reaction (or propagation) may be terminated by formation of non-radical products resulting from combination of two radicals species (Reactions 4 to 6).

Initiation:

$$
\mathrm{RH} \stackrel{\text { initiator }}{\longrightarrow} \mathrm{R}^{\cdot}+\mathrm{H}^{\cdot}
$$

Propagation:

$$
\begin{aligned}
& \mathrm{R}^{\bullet}+\mathrm{O}_{2} \rightarrow \mathrm{ROO}^{\bullet} \\
& \mathrm{ROO}^{*}+\mathrm{RH} \rightarrow \mathrm{ROOH}+\mathrm{R}^{\bullet}
\end{aligned}
$$

Termination:

$$
\begin{gathered}
\mathrm{R}^{\cdot}+\mathrm{R}^{\cdot} \rightarrow \mathrm{RR} \\
\mathrm{R}^{*}+\mathrm{ROO}^{\cdot} \rightarrow \mathrm{ROOR} \\
\mathrm{ROO}^{*}+\mathrm{ROO}^{\cdot} \rightarrow \mathrm{ROOR}+\mathrm{O}_{2}
\end{gathered}
$$

The mechanism of lipid autoxidation has been postulated by Farmer et al. (1942), Boland and Gee (1946) and Bateman et al. (1953). The propagation step in the autoxidation process includes an induction period when hydroperoxide formation is minimal (Labuza, 1971; Hawrysh, 1990). The rate of oxidation of fatty acids depends on the degree of unsaturation; the relative rate of autoxidation of oleate, linoleate and linolenate is in the order of $1: 40-50: 100$, on the basis of oxygen uptake, and $1: 12: 25$, on the basis of peroxide formation (Hsieh \& Kinsella, 1989). Polyunsaturated fatty acids (PUFA) such as arachidonic acid, eicosapentaenoic acid (EPA) and docosahexaenoic acid (DHA), containing 4, 5 and 6 double bonds, respectively, are much less stable than linoleic and linolenic acids. Arachidonic acid is oxidized 2.9 times faster than linoleic acid (Porter et al, 1981). Ethyl esters of EPA and DHA oxidized rapidly even at $5^{\circ} \mathrm{C}$ in the dark after an induction period of 3-4 days, whereas the induction period of linoleate and linolenate under similar conditions are 20 and 60 days, respectively. Therefore, oils that contain relatively high amounts of PUFA experience stability problems. The breakdown products of hydroperoxides such as alcohols, aldehydes, ketones and hydrocarbons generally possess offensive off-flavors. These compounds may also interact with other food components and bring about changes in their functional and nutritional properties (Sherwin, 1978). In addition, free radicals formed 


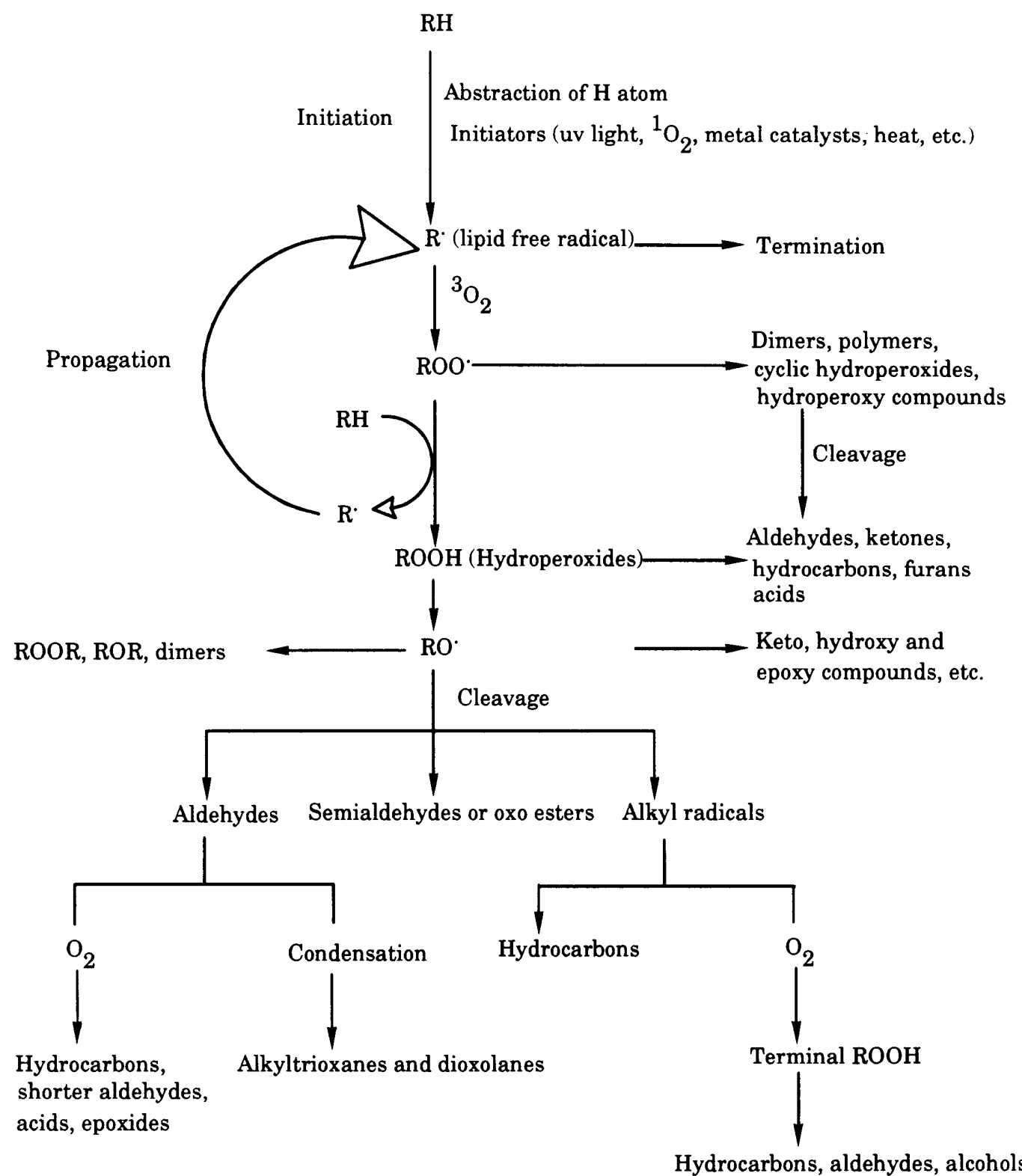

Fig. 1. General scheme for autoxidation of food lipids and its possible products

during oxidation of lipids may cause damage to membranes, enzymes, vitamins and proteins and may also be involved in the process of ageing (Hamilton, 1983). Autoxidation of food-lipids and its possible products are illustrated in Fig. 1.

\section{Measurement of Oxidative Stability of Food Lipids}

There are various methods available to measure lipid oxidation in foods. Changes in chemical, physical, or organoleptic properties of fats and oils during oxidation may be monitored in order to assess the extent of lipid oxidation. However, there is no uniform and standard method for detecting all oxidative changes in all food systems. The available methods to monitor lipid oxidation in foods and biological systems may be divided into two groups. The first group measures primary changes and the second group measures secondary changes that occur in each system. Primary changes are generally measured by monitoring (a) loss of unsaturated fatty acids, (b) oxygen uptake by weight gain or other methods, (c) hydroperoxide values and (d) conjugated diene value. Secondary changes are followed by quantitation of (a) carbonyl compounds (as their dinitrophenylhydrazone or by gas chromatography), (b) malonaldehyde and other aldehydes (TBA test), (c) hydrocarbons (ethane or pentane content) and (d) fluorescence products (1-amino-3-iminopropene derivatives). The method of choice depends on a number of factors including the nature and the history of the oxidized sample, the type of information required, the time available, and the test conditions. Nonetheless, it is advisable to use a combination of methods to assess both primary and secondary oxidative changes in lipids. However, the results must be in agreement with sensory perception and acceptability standards for foods.

Primary changes

Changes in reactants Methods that measure primary 
changes of lipids may be classified as those that quantify loss of reactants (unsaturated fatty acids), addition of oxygen or formation of primary lipid oxidation products (hydroperoxides). These methods are more suitable to measure low levels of oxidation in uncooked products at low temperatures (Coxon, 1987). Measurement of changes in fatty acid composition is not widely used in assessing lipid oxidation since it requires total lipid extraction and conversion into derivatives suitable for gas chromatographic analysis; separation of lipids into neutral, glycolipid, phospholipid and other classes may also be necessary. However, it has been proven that this method serves as a useful technique to identify which class of lipids and fatty acids is involved in the oxidative changes (Coxon, 1987; Gray \& Monahan, 1992) and also to assess lipid oxidation simulated by different metal complexes that give different products (Gutteridge \& Halliwell, 1990). On the other hand, changes of fatty acid composition can not be used in more staturated oils since this indicator reflects only the changes that occur in unsaturated fatty acids during oxidation. Therefore, oxidative changes in marine oils and highly unsaturated vegetable oils may be monitored using this indicator. Similarly, changes in iodine value (IV) due to loss of unsaturation may be employed to monitor oxidation of food lipids (Hudson, 1983).

During oxidation the oxygen above the lipid surface slowly reacts with lipids and changes in the oxygen pressure may be measured quantitatively. This is the principle used in the Sylvester test in which the oil is subjected to a temperatures of $100^{\circ} \mathrm{C}$ in a closed system. The drop in pressure of the system is quantified as oxidation proceeds.

Weight gain It is generally accepted that addition of oxygen to lipids and formation of hydroperoxides is reasonably quantitative during initial stages of autoxidation. Therefore, the measurement of induction period from weight gain data is theoretically sound. In this method oil samples (about $2.0 \mathrm{~g}$ ) are weighed into Petri dishes; traces of water are removed by placing them overnight in a vacuum oven at $35^{\circ} \mathrm{C}$ and over a desiccant. The samples are then reweighed and stored in an oven at a set temperature. The weight gain of the samples may be recorded at different time intervals.

Olcott and Einest (1958) reported that marine oils exhibit a fairly sharp increase in mass by the end of the induction period and are rancid by the time they gain $0.3-0.5 \%$ in weight (at $30-60^{\circ} \mathrm{C}$ ). Ke \& Ackman (1976) reported that this method is simple, has a satisfactory reproducibility and may be used to compare oxidation of lipids from different parts of fish. Recently, Wanasundara \& Shahidi (1994) and Shahidi et al. (1994) used this method to monitor storage stability of both vegetable and marine oils as such, and as affected by added natural and synthetic antioxidants. Relative activity of different antioxidants may also be compared in this manner. However, surface exposure of the sample to air is an important variable in determining the rate of oxidation. Therefore, use of equal size containers to store samples is very important in carrying out such experiments.

The weight-gain method also suffers from certain disadvantages: (1) the weighing frequency hinders monitoring of fast kinetics (a higher frequency would involve nocturnal weighing), and low or moderate temperatures require long analysis times for stable samples, (2) discontinuous heating of the sample (which must be cooled before weighing) may give rise to irreproducible results, so the heating and cooling intervals must be accurately controlled, (3) the method involves intensive human participation and (4) the working conditions (sample size, shape of the container and temperature) influence the results. Nevertheless, this method offers advantages such as low instrumentation cost and unlimited capacity for sample processing.

Hydroperoxides In the oxidation of fats and oils, the initial rate of formation of hydroperoxides exceeds their rate of decomposition, but this is reversed at later stages. Therefore, monitoring the amount of hydroperoxides as a function of time will indicate whether a lipid is in the growth or decay portion of the hydroperoxide concentration curve. This information can be used as a guide for considering the acceptability of a food product with respect to the extent of product deterioration. By monitoring the induction period before the appearance of hydroperoxides, one can assess the effectiveness of added antioxidants on the stability of a food lipid.

Peroxide value The classical method for quantitation of hydroperoxides is the determination of "peroxide value" (PV). The hydroperoxide content, generally referred to as peroxide content, is determined by an iodometric method. This is based on the titration of the iodine released from potassium iodide by the peroxides in a biphasic system with a thiosulfate solution as given below.

$$
\begin{aligned}
& \mathrm{ROOH}+2 \mathrm{H}^{+}+2 \mathrm{I}^{-} \rightarrow \mathrm{I}_{2}+\mathrm{ROH}+\mathrm{H}_{2} \mathrm{O} \\
& \mathrm{I}_{2}+2 \mathrm{~S}_{2} \mathrm{O}_{3}{ }^{2+} \rightarrow \mathrm{S}_{4} \mathrm{O}_{6}{ }^{2+}+2 \mathrm{I}^{-}
\end{aligned}
$$

Potential drawbacks of this method are absorption of iodine at unsaturation sites of fatty acids and liberation of iodine from potassium iodide by oxygen present in the solution to be titrated (Gray, 1978). Results may also be affected by the structure and reactivity of peroxides as well as reaction temperature and time. The official iodometric method Cd 8-53 (AOCS, 1990) for determination of PV is applicable to all normal fats and oils, but is highly empirical and any variation in procedure may result in variation in the results. The official method also fails to adequately measure low PV because of difficulties for determination of the titration end point. Therefore, the official method has been modified in an attempt to increase sensitivity for determination of low PV. The modification involves the replacement of the titration step with an electrochemical technique in which the liberated iodine is reduced at a platinum electrode maintained at a constant potential with this method. PV ranging from 0.06 to 20 have been determined with this method, but it is essential to deaerate all solutions in order to prevent further formation of peroxides.

Several other chemical methods have also been suggested for monitoring PV. Colorimetric methods based on the oxidation of $\mathrm{Fe}^{2+}$ to $\mathrm{Fe}^{3+}$ and determination of $\mathrm{Fe}^{3+}$ as ferric thiocyanate, and a 2,6-dichlorophenol-indophenol procedure are reported in the literature (Lips et al. 1943). In studies on the oxidation of biological tissues and fluids, measurement of fatty acid hydroperoxides is more common than measuring their decomposition products. Fatty acid hydroperoxides can 
be analyzed by high performance liquid chromatography (HPLC), or their corresponding hydroperoxy acid reduction products can be determined by gas chromatography-mass spectrometry (GC-MS) (Hughes et al., 1983, 1986). Fluorescence methods have also been developed to determine hydroperoxides by allowing them to react with substances such as luminol and dichlorofluorescein which form fluorescent products (Gray, 1978). Although determination of peroxide value is common, its usefulness is generally limited to the initial stages of lipid oxidation.

Active oxygen and oil stability index/Rancimat/Oxidative Stability Instrument (OSI) methods The Active Oxygen Method (AOM), also referred to as the Swift test of the American Oil Chemist's Society, is a commonly used accelerated method for assessing oxidative stability of fats and oils. This method is based on the principle that aging and rancidification of a fat is greatly accelerated by aeration in a tube held at a constant elevated temperature. The peroxide value reached by the AOM at which a fat will be rancid by organoleptic evaluation varies with the nature of the fat (Dugan, 1955). Even though this method has been used extensively over the years, its inherent deficiencies and difficulties also have been identified; for example, (1) the end point is determined by the amount of peroxides in the oxidized oil and peroxides are unstable and decompose readily to more stable secondary products, (2) during the rapid oxidation phase, the reaction is extremely susceptible to variation in the oxygen supply.

Automated versions of the AOM apparatus, known as the Oil Stability Instrument (OSI) and Rancimat, are now available. The Rancimat method uses a commercial apparatus marketed by Metrohm, Ltd. The OSI, computer-assisted version developed by Archer-Daniels-Midland Co. (ADM) is now produced commercially by Omnion Inc. (Rockland, MA). These methods may be considered as automated AOM since both employ the principle of accelerated oxidation. However, the OSI and Rancimat tests measured the changes in conductivity caused by ionic volatile organic acids, mainly formic acid, automatically and continuously, whereas in the AOM, peroxide values are measured. These organic acids are stable oxidation reaction products which are produced when oil is oxidized by a stream of air bubbled through it. As in the AOM, the OSI or Rancimat tests proceed slowly at first because during the induction period, little acid is released. The end point is selected when the rapid rise in conductance begins. The Rancimat became available in the early 1980s and is capable of running only six samples at the same time, whereas the OSI is capable of running twenty-four samples at the same time.

Jebe et al. (1993) have reported that the newly developed OSI method is superior over the AOM in many respects: (a) in the AOM, the end point does not determine the induction period, but is merely related to it, whereas the OSI end point determines the induction period precisely, (b) the AOM provides a single data point, whereas OSI provides continual data points, (c) the AOM relies on analysis of unstable primary reaction products, while the OSI is based on analysis of stable secondary products, (d) in the AOM, the end point is determined during the oxidant dependent, highly variable and difficult-to-reproduce rapid oxidation phase, whereas the OSI end point is determined at the end of the induction period, which is relatively insensitive to oxidant flow, (e) the AOM depends on a manually obtained end point, whereas OSI has an instrumental automatic end point and (f) the AOM is manual, and labor-intensive and time-consuming, but OSI is automated. Despite the high cost of these apparatus, OSI and Rancimat are currently regarded as the methods of choice for fat stability studies by the industry.

Conjugated dienes Oxidation of polyunsaturated fatty acids is accompanied by an increase in the ultraviolet absorption of the product. Lipids containing methyleneinterrupted dienes or polyenes show a shift in their double bond position during oxidation due to isomerization and conjugation formation (Logani \& Davies, 1980). The resulting conjugated dienes exhibit intense absorption at $234 \mathrm{~nm}$; similarly, conjugated trienes absorb at $268 \mathrm{~nm}$. Farmer and Sutton (1946) indicated that the absorption increase due to the formation of conjugated dienes and trienes is proportional to the uptake of oxygen and formation of peroxides during the early stages of oxidation. St. Angelo et al. (1972; 1975) studied the autoxidation of peanut butter by measuring the PV and absorption increase at $234 \mathrm{~nm}$ due to the formation of conjugated dienes. Shahidi et al. (1994) and Wanasundara et al. (1995a) have found that conjugated dienes (CD) correlate with peroxide values during the oxidation of marine (seal blubber and cod liver) and vegetable (canola and soybean) oils. These authors concluded that the CD method may be used as an index of stability of lipids in place of, or in addition to PV. However, carotenoidcontaining oils may give high absorbance values at 234-236 $\mathrm{nm}$ due to the presence of double bonds in their conjugated structures and may therefore, give erroneous results. The CD method is faster than the PV determination, is much simpler, does not depend upon chemical reaction or color development, and requires a smaller sample size. However, existence of a linear relationship between PV and CD may not be expected for all oils and the latter method is especially suitable when extraction is not desirable.

Parr \& Swoboda (1976) have described another spectroscopic method to determine lipid oxidation of stored oils. In this assay, hydroperoxides of polyenoic fatty acids as well as hydroxy and carbonyl compounds derived from them are converted to more conjugated chromophores by two chemical reaction steps, first reduction and then dehydration. These yield "conjugable oxidation products (COP)" which are measured and expressed as the COP value. The first step of the analytical procedure, reduction with sodium or sodium borohydride, results in the disapprearance of the characteristic ultraviolet absorption of the carbonyl compounds of oxidized polyenoic fatty acids (oxodienes). The decrease in absorption at $275 \mathrm{~nm}$ is known as the oxodiene value. Another feature of this assay is that it distinguishes between the oxidation products derived from dienoic and polyenoic fatty acids as measured by the COP ratio.

Secondary changes The primary oxidation products (peroxides) of fats and oils are transitionary intermediates that decompose into various secondary products. Measurement of secondary oxidation products as an index of lipid 
oxidation is more appropriate since secondary products of oxidation are generally flavor-active, whereas primary oxidation products are colorless and flavorless. Secondary oxidation products include ketones, aldehydes, hydrocarbons and alcohols, among others.

2-Thiobarbituric acid (TBA) value One of the oldest and most frequently used tests for assessing lipid oxidation in foods and other biological systems is the 2-thiobarbituric acid (TBA) test. The extent of lipid oxidation is reported as the TBA value and is expressed as milligrams of malonaldehyde (MA) equivalents per kilogram sample or as $\mu$ mole MA equivalents/g sample. Malonaldehyde is a relatively minor product of oxidation of polyunsaturated fatty acids which reacts with the TBA reagent to produce a pink-colored complex with an absorption maximum at $530-532 \mathrm{~nm}$ (Tarladgis et al., 1964). The adduct is formed by condensation of $2 \mathrm{~mol}$ of TBA with $1 \mathrm{~mol}$ of MA as given above.

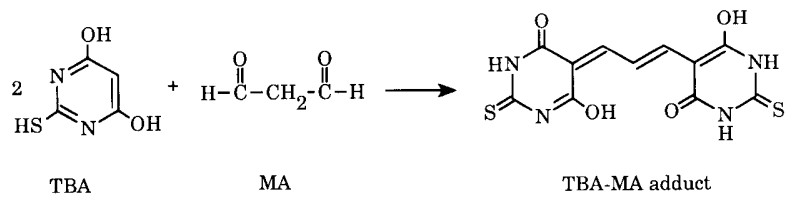

Dahle et al. (1962) postulated a mechanism for the formation of MA and indicated that only peroxides which possess unsaturation $\beta$ or $\gamma$ to the peroxide group are capable of undergoing cyclization with the ultimate formation of MA. Such peroxides may be produced from fatty acids containing three or more double bonds (Dahle et al., 1962). Other products of lipid oxidation, such as aldehydes, alkenals and other dienals (alka-2,4-dienals) may also react with the TBA reagent to form a pink-colored complex with the same absorption maximum as the MA-TBA complex (Marcuse \& Johansson, 1973). Therefore, the term "thiobarbituric acidreactive substances" (TBARS) is now commonly used in place of TBA value (Shahidi et al., 1985; Ke et al., 1984; Wanasundara \& Shahidi, 1994).

There are certain limitations when using the TBA test for evaluation of the oxidative state of foods and biological systems because of the chemical complexity of these systems. Dugan (1955) has reported that sucrose and some compounds in woodsmoke react with the TBA reagent to give a red color interfering with the TBA test. Baumgartner et al. (1975) have also found that a mixture of acetaldehyde and sucrose when subjected to the TBA test produced a $532 \mathrm{~nm}$ absorbing pigment identical to that produced by MA and TBA. Modifications of the original TBA test have been reported by Marcuse \& Johansson (1973), Ke \& Woyewoda (1979), Robles-Martinez et al. (1982), Pokorny et al. (1985), Shahidi et al. (1987), Tomas \& Funes (1987) and Schmedes \& Holmer (1989). However, it has been suggested TBARS provide an excellent means for evaluating the relative oxidative status of a system as affected by storage or processing variables (Gray \& Pearson, 1987). It is always preferable to quantitate the extent of lipid oxidation by a complementary and analytical procedure to verify the results.

Several attempts have been made to establish a relationship between TBA values and the development of undesirable

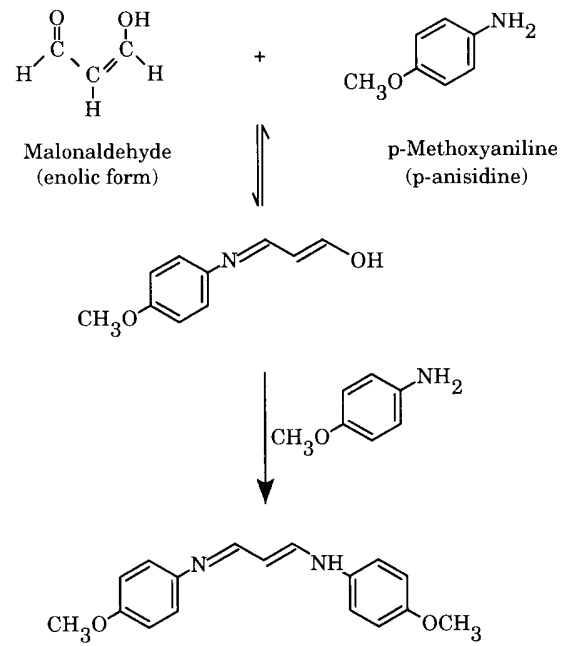

Fig. 2. Possible reactions between p-anisidine and malonaldehyde.

flavors in fats and oils. It has been shown that flavor threshold values correlate well with TBA values of vegetable oils such as those of soybean, cottonseed, corn, safflower (Gray, 1978) and canola (Hawrysh, 1990).

Oxirane value The oxirane oxygen groups, also known as the epoxide groups, are formed during autoxidation of fats and oils. The epoxide content is determined by titrating the oil sample with $\mathrm{HBr}$ in acetic acid, in the presence of crystal violet, to a bluish-green end point. This method has been standardized by the American Oil Chemists' Society in their tentative methods Cd 9-57 (1981). However, this test is not sensitive and lacks specificity. $\mathrm{HBr}$ also attacks $\alpha, \beta$-unsaturated carbonyls and conjugated dienols and this reaction is not quantitative with some trans-epoxides. Picric acid was the best of several acidic chromophores in its reaction with epoxides. Despite a non-quantitative reaction, the product concentration followed Beer's Law. This method has been found to be particularly well suited to the determination of epoxides in heated fats where the oxirane content is often less than $0.1 \%$.

$p$-Anisidine value $p$-Anisidine value is used to measure secondary products of lipid oxidation. This method determines the amount of aldehydes (principally 2-alkenals and alka-2,4-dienals) in animal fats and vegetable oils. Aldehydes in an oil and the $p$-anisidine reagent react under acidic conditions (IUPAC, 1987; method number 2,504). The proposed reaction of $p$-anisidine with aldehydes to form yellowish products is shown in Fig. 2. List et al. (1974) have reported a highly significant correlation between $p$-anisidine values of salad oils processed from undamaged soybeans and their flavor acceptability scores.

TOTOX value The $p$-anisidine value is often used in the industry in conjunction with PV to calculate the so-called total oxidation or TOTOX value:

TOTOX value $=2 \mathrm{PV}+p$-Anisidine value

The TOTOX value is often considered to have the advantage of combining evidence about the past history of an oil (as reflected in the $p$-anisidine value) with its present state 
(as evidenced in the PV). Therefore, determination of TOTOX value has been used extensively to estimate oxidative deterioration of food lipids (Rossell, 1983). However, despite its practical advantages, the TOTOX value does not have any sound scientific basis since it combines variables with different dimensions. Recently, Wanasundara and Shahidi (1995a) have defined TOTOX $_{\mathrm{TBA}}$ as $2 \mathrm{PV}+\mathrm{TBARS}$ since measuring of $p$-anisidine may not always be as feasible as that of TBARS.

Carbonyls An alternative approach for monitoring the extent of lipid oxidation in fats and oils is to measure the total or individual volatile carbonyl compounds formed from degradation of hydroperoxides (Shahidi et al., 1986). One of the more reliable methods for total carbonyl analysis is based on the absorbance of the quinoidal ion, a derivative of aldehydes and ketones. This ion is formed from the reaction of 2,4-dinitrophenylhydrazine (2,4-DNPH) with an aldehyde or ketone, followed by the reaction of the resulting hydrozones with alkali as given above. Many variations of this spectroscopic method have been reported (Yukawa et al., 1993; Meyer \& Rebrovic, 1995). Each method offers an alternative solvent, wavelength, or workup in which to analyze the quinoidal ion.

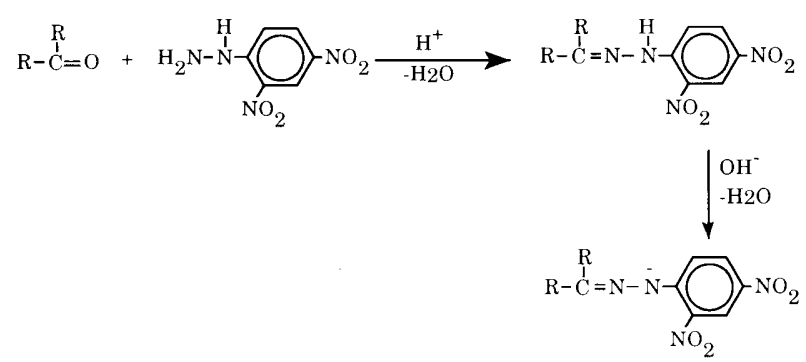

Hexanal, one of the major secondary products formed during the oxidation of linoleic acid in fats and oils (Frankel et al., 1981; Shahidi \& Pegg, 1994), and other aldehydes have been used to follow lipid oxidation in meat products. Shahidi et al. $(1987$; 1991) reported a linear relationship between hexanal content, sensory scores and TBA numbers of cooked ground pork, while St. Angelo et al. (1987) established a similar correlation for cooked beef. Recent studies in our laboratories have shown that during oxidation of oils rich in polyunsaturated fatty acids of the omega-3 type, large amounts of propanal are fomed (Frankel, 1993) and a good correlation existed between the propanal content and TBARS values (Wanasundara \& Shahidi, 1995b). These studies suggested that compounds usually associated with lipid oxidation could be used as marker compounds to follow development of off-flavors in lipid-containing foods.

Hydrocarbons and fluorescent products Oxidative studies with methyl linoleate and soybean oil (Selke et al., 1970) have revealed that saturated hydrocarbons could be detected when aldehydes are either absent or undetectable. Snyder et al. (1985) have reported that ethane, propane and pentane are the predominant short-chain hydrocarbons formed through thermal decomposition of soybean oil. Correlations between flavor acceptability scores and pentane content, as determined by gas chromatographic techniques, have been used to assess rancidity of fats and oils (Jarvi et al., 1971). Significant correlations existed between the amount of pentane produced and the number of rancid descriptions of aged vegetable oils (Warner et al., 1974). Headspace pentane concentrations and sensory scores for rancidity development in stored freeze-dried pork samples were reported by Coxon (1987).

Another secondary change that occurs during autoxidation of biological systems is the formation of fluorescent products from the reaction of $\mathrm{MA}$ with amino compounds such as proteins and nucleotides (Bidlack \& Tappel, 1973). This method has been used in determining the extent of lipid oxidation in biological tissues. Dillard \& Tappel (1973) have established that fluorescent compounds with the general structure of a 1-amino-3-iminopropene may develop through the reaction of an amino group with carbonyl compounds, mainly malonaldehyde:

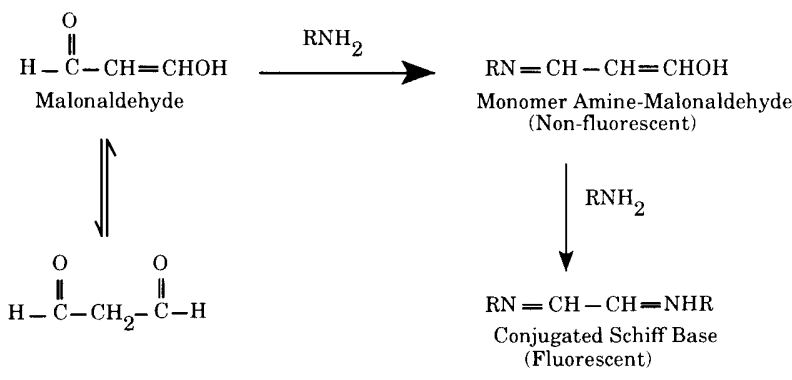

Gray (1978) reported that the development of fluorescent compounds depends not only on the formation of condensation products between malonaldehyde and free amino group but also on the nature of the substituents of the amino groups. Different excitation and emission maxima were observed for different condensation products. Advantages of the fluorescent compounds method as a means of measuring lipid oxidation have been reported by Dillard \& Tappel (1971; 1973). This method can detect fluorescent compounds at a parts per billion level and was found to be 10 to 100 times more sensitive than the TBA assay.

\section{Recent Developments for Quantitation of Lipid Oxidation}

Lipid oxidation in foods and biological systems has conventionally been studied by monitoring either primary or secondary oxidation products. Over the last twenty years or so advances in pulse radiolysis (Simic, 1980) and electron spin resonance (ESR; Schnaich and Borgi, 1980) techniques have facilitated the detection and study of short-lived free radical intermediates. ESR spectroscopy selectively detects species with an unpaired electron, such as free radicals. The technique depends on the absorption of microwave energy (which arises from the promotion of an electron to a higher energy level) when a sample is placed in a variable magnetic field. A major limitation in the detection of free radicals by ESR is the requirement that radical concentrations remain higher than $10^{-8} \mathrm{M}$. Radical lifetimes in solution are very short $(<1 \mathrm{~ms})$ and steady state concentrations generally remain well below $10^{-7} \mathrm{M}$. Several approaches have been developed to overcome this problem, based on either enhancing the rate of radical production or diminishing the rate of its disappearance. 
These techniques include rapid freezing, lyophilization or spin trapping (Davies, 1987). Although application of ESR as a precise method to study lipid oxidation in animal tissues and other biological model systems is commonplace, its application to food systems is relatively new.

Recently, Burkow et al. (1992) have reported that hypochlorite-activated chemiluminescence could provide a useful method for evaluation of antioxidants in edible oils. Due to high sensitivity and ability to detect small changes in the degree of oxidation, this method provides a useful means to evaluate effect of antioxidants on oils at low temperature (about $35^{\circ} \mathrm{C}$ ) storage within $24 \mathrm{~h}$. Chemiluminescence generally originates from electrically excited stages, such as singlet molecular oxygen in lipid peroxidation (Neeman et al., 1985). The chemiluminescent method has now been tested for estimating the deterioration of edible oils containing antioxidants (Burkow et al., 1995), as well as for shelf-life dating of fish and meats (Miyazawa et al., 1991).

Infrared (IR) spectroscopy has also been used for the measurement of rancidity, and it is of particular value in the recognition of unusual functional groups and in the study of fatty acids with trans double bonds. Production of hydroperoxides during oxidation of lipids gives rise to a band at about $2.93 \mu \mathrm{m}$ whereas the disappearance of a band at about 3.20 $\mu \mathrm{m}$ indicates the replacement of a hydrogen on a double bond, or polymerization. It is also suggested that the appearance of an additional band at $5.72 \mu \mathrm{m}$, due to $\mathrm{C}=\mathrm{O}$ stretching, indicates the formation of aldehydes, ketones or acids. Furthermore, changes in the absorption bands in the 10 to 11 $\mu \mathrm{m}$ region indicates cis, trans-isomerizations and formation of conjugated ethylenic bonds. Recently, van de Voort et al. (1994) have reported that Fourier Transform Infrared (FTIR) spectroscopy could provide a rapid measurement of oxidative deterioration of lipids quantitatively by percent hydroperoxides, percent alcohols and total carbonyl content.

Determination of overall changes in the oxidative state of food lipids including both primary and secondary changes remains desirable (Shahidi, 1992). During oxidation of food lipids, changes occur to the protons in an oxidizing molecule. These changes may be monitored by employing proton Nuclear Magnetic Resonance ( ${ }^{1} \mathrm{H}$ NMR) spectroscopy as originally described by Saito \& Udagawa (1992) and Wanasundara \& Shahidi (1993). During oxidation of lipids with unsaturated fatty acid, the relative number of olefinic and diallylmethylene protons decreases and the total number of aliphatic protons increases. Diallylmethylene protons are first attacked by free radicals and intramolecular rearragement of the olefinic protons would follows. Thus, the relative number of diallylmethylene and olefinic protons decreases. NMR absorption peaks of diallylmethylene, olefinic and aliphatic protons appear at $\delta 2.6-2.9, \delta 5.1-5.6$ and $\delta 0.6-2.5$, respectively (Saito \& Udagawa, 1992) Wanasundara \& Shahidi (1993) have shown that the ratios of aliphatic to olefinic protons $\left(R_{\mathrm{ao}}\right)$ and aliphatic to diallylmethylene protons $\left(R_{\mathrm{ad}}\right)$ increase steadily during the oxidation of canola and soybean oils. Similar results were observed for both cod liver and seal blubber oils (Shahidi et al, 1994). Furthermore, plotting of $R_{\text {ao }}$ and $R_{\text {ad }}$ against corresponding TOTOX values of these oils indicated significant $(p<0.05)$ linear correlations. Therefore, the NMR methodology offers an alternative method to estimate the overall changes in the primary and secondary oxidation products of lipids. It also provides a rapid, nondestructive and simple procedure for evaluation of the oxidative changes during storage of lipids.

\section{Methods of Assessing and Screening of Antioxidant Activity}

Methods described above might also be used for scrutinizing the activity of antioxidants or comparing their effectiveness with other antioxidants. However, there are other methods such as Fenton reaction (Mason et al, 1990), free radical scavenging determination (Brand-William et al, 1995), $\beta$ - carotene bleaching (Miller, 1971) and hemoglobin peroxidation test (Cort, 1974) which may also be used to compare oxidative stability of lipids or screening of new antioxidants.

\section{References}

American Oil Chemists' Society (1989). "Official Methods and Recommended Practices of the American Oil Chemists' Society," 4th Ed., ed. by D. Firestone. American Oil Chemists' Society, Champaign, IL.

Bateman, L., Hughes, H. and Morris, A.L. (1953). Hydroperoxide decomposition in relation to the initiation of radical chain reactions. Discuss. Faraday Soc., 14, 190-194.

Baumgartner, W.A., Baker, N., Hill, V.A. and Wright, E.T. (1975). Novel interference in thiobarbituric acid assay for lipid peroxidation. Lipids, 10, 309-311.

Bidlack, W.R. and Tappel, A.L. (1973). Fluorescent products of phospholipids during lipid peroxidation. Lipids, 8, 203-207.

Boland, J.L. and Gee, G. (1946). Kinetics in the chemistry of rubber and related materials. Trans. Faraday Soc., 42, 236-243.

Brand-Williams, W., Cuvelier, M.E. and Berset, C. (1995). Use of free radical method to evaluate antioxidant activity. Lebensm. Wiss. Technol., 28, 25-30.

Burkow, I.C., Moen, P. and Overbo, K. (1992). Chemiluminescence as a method for oxidative rancidity assessment in autoxidized marine oils. J. Am. Oil Chem. Soc., 69, 1108-1111.

Burkow, I.C., Vikersveen, L. and Saarem, K. (1995). Evaluation of antioxidants for cod liver oil by chemiluminescence and the rancimat method. J. Am. Oil Chem. Soc., 72, 553-557.

Cort, W.M. (1974). Hemoglobin peroxidation test screens antioxidants. Food Technol., 28(10), 60-66.

Coxon, D. (1987). Measurement of lipid oxidation. Food Sci. Technol. Today, 1, 164-166

Dahle, L.K., Hill, E.G. and Holman, R.T. (1962). The thiobarbituric acid reaction and autoxidation of polyunsaturated fatty acids. Arch. Biochem. Biophys., 98, 253-261.

Davies, M.J. (1987). Application of electron spin resonance spectroscopy to the identification of radicals produced during lipid peroxidation. Chem. Phys. Lipids, 44, 149-173.

Dillard, C.J. and Tappel, A.L. (1971). Fluorescent products of lipid peroxidation of mitochondria and microsomes. Lipids, 6, 715-721.

Dillard, C.J. and Tappel, A.L. (1973). Fluorescent products from reaction of peroxidizing polyunsaturated fatty acids with phosphatidyl ethanolamine and phenylalanine. Lipids, 8, 183-189.

Dugan, L.R. Jr. (1955). Stability and rancidity. J. Am. Oil Chem. Soc., 32, 605-609.

Farmer, E.H., Bloomfield, G.F., Sundaralingam, A. and Sutton, D.A. (1942). The course and mechanism of autoxidation reactions in olefinic and polyolefinic substances including rubber. Trans. Faraday Soc., 38, 348-356.

Farmer, E.H. and Sutton, D.A. (1946). Peroxidation in relation to olefinic structure. Trans. Faraday Soc., 42, 228-232.

Frankel, E.N. (1980). Lipid oxidation: A review. Prog. Lipid Res., 19, $1-22$. 
Frankel, E.N. (1993). Formation of headspace volatiles by thermal decomposition of oxidized fish oil vs. oxidized vegetable oils. J. Am. Oil Chem. Soc, , 70, 767-772.

Frankel, E.N., Neff, W.E. and Selke, E. (1981). Analysis of autoxidized fats by gas chromatography-mass spectrometry: VII. Volatile thermal decomposition products of pure hydroperoxides from autoxidized and photosensitized oxidized methyl oleate, linoleate and linolenate. Lipids, 16, 279-285.

Gray, J.I. (1978). Measurement of lipid oxidation: A review. J. Am. Oil Chem. Soc., 55, 539-546.

Gray, J.I. and Pearson, A.M. (1987). Rancidity and warmed-over flavor. In "Advances in Meat Research," Vol. 3: Restructured Meat and Poultry products, ed. by A.M. Pearson and T.R. Dutson. Van Nostrand Reinhold, NY, pp. 221-269.

Gray, J.I. and Monahan, F.J. (1992). Measurement of lipid oxidation in meat and meat products. Trends Food Sci. Technol., 3, 320-324.

Gutteridge, J.M.C. and Halliwell, B. (1990). The measurement and mechanism of lipid peroxidation in biological systems. Trends Biochem. Sci., 15, 129-135.

Hamilton, R.J. (1983). The chemistry of rancidity in foods. In "Rancidity in Foods," ed. by J.C. Allen and R.J. Hamilton. Applied Science Publishers, NY, pp. 1-20.

Hawrysh, Z.J. (1990). Stability of canola oil. In "Canola and Rapeseed, Production, Chemistry, Nutrition and Processing Technology," ed. by F. Shahidi. Van Nostrand Reinhold, NY, pp. 99122.

Horton, A.A. and Fairhurst, S. (1987). Lipid peroxidation and mechanisms of toxicity. CRC Crit. Rev. Toxicol., 18, 27-29.

Hsieh, R.J. and Kinsella, J.E. (1989). Oxidation of polyunsaturated fatty acids: mechanisms, products and inhibition with emphasis on fish. Adv. Food Nutr. Res., 33, 233-24l.

Hudson, B.J.F. (1983). Evaluation of oxidative rancidity technique. In "Rancidity of Foods," ed. by J.C. Allen and J. Hamilton. Applied Science. Publishers, London, pp. 47-58.

Hughes, H., Smith, C.V., Horning, E.C. and Mitchell, J.R. (1983). High-performance liquid chromatography and gas chromatography-mass spectrometry determinations of specific lipid peroxidation products, In Vivo. Anal. Biochem., 130, 431-436.

Hughes, H., Smith, C.V., Tsokos-Kuhn, J.O. and Mitchell, J.R. (1986). Quantification of lipid peroxidation products by gas chromatography-mass spectrometry. Anal. Biochem., 152, 107-112.

IUPAC (1987). "Standard Methods for the Analysis of Oils and Fats and Derivatives," 7th Ed. Blackwell Scientific Publ. Oxford.

Jarvi, P.K., Lee, G.D., Erickson, D.K. and Butkus, E.A. (1971). Determination of the extent of rancidity of soybean oil by gas chromatography compared with peroxide values. J. Am. Oil Chem. Soc., 48, $121-124$.

Jebe, T.A., Matlock, M.G. and Sleeter, R.T. (1993). Collaborative study of the oil stability index analysis. J. Am. Oil Chem. Soc., 70, 1055-1061.

Kanner, J., German, J.B. and Kinsella, J.E. (1987). Initiation of lipid peroxidation in biological systems. CRC Crit. Rev. Food Sci. Nutr., 25, 317-364.

Ke, P.J. and Ackman, R.G. (1976). Metal-catalyzed oxidation in mackerel skin and meat lipids. J. Am. Oil Chem. Soc., 53, 636-640.

Ke, P.J. and Woyewoda, A.D. (1979). Microdetermination of thiobarbituric acid values in marine lipids by a direct spectrophotometric method with a monophasic reaction system. Anal. Chim. Acta, 106, 279-284.

Ke, P.J., Cervantes, E. and Robles-Martinez, C. (1984). Determination of thiobarbituric acid reactive substances (TBARS) in fish tissue by an improved distillation-spectrophotometric method. J. Sci. Food Agric., 35, 1248-1254.

Labuza, T.P. (1971). Kinetics of lipid oxidation in foods: A review. CRC Crit. Rev. Food Technol., 2, 355-405.

Lips, A., Chapman, R.A. and McFarlane, W.D. (1943). The application of ferric thiocyanate method to the determination of incipient rancidity in fats and oils. Oil Soap, 20, 240-243.

List, G.R., Evans, C.D., Kwolek, W.K., Warner, K. and Boundy, B.K (1974). Oxidation and quality of soybean oil: A preliminary study of the anisidine test. J.Am. Oil Chem. Soc., 51, 17-21.
Logani, M.K. and Davies, R.E. (1980). Lipid oxidation: Biological effects and antioxidants: A review. Lipids, 15, 485-495.

Marcuse, R. and Johansson, L. (1973). Studies on the TBA test for rancidity grading: II. TBA reactivity of different aldehyde classes. $J$. Am. Oil Chem. Soc., 50, 387-391.

Mason, R.P., Stolze, K. and Flitter, W.D. (1990). Free radical reaction with DNA and its nucleotides. In "Antimutagenesis and Anticarcinogenesis Mechanisms II," ed. by Y. Kuroda, D.M. Shankel and M.D. Waters. Plenum Press, NY, pp. 119-125.

Meyer, S.R. and Rebrovic, L. (1995). The spectroscopic quinodal ion method for the analysis of carbonyl compounds. J. Am. Oil Chem. Soc., 72, 385-387

Miller, H.E. (1971). A simplified method for the evaluation of antioxidants. J. Am. Oil Chem. Soc., 44, 91.

Miyazawa, T., Kikuchi, M., Fujimoto, K., Endo, Y., Cho, S.Y., Usuki, R. and Kaneda, T. (1991). Shelf-life dating of fish meats in terms of oxidative rancidity as measured by chemiluminescence. $\mathrm{J}$. Am. Oil Chem. Soc., 68, 39-43.

Neeman, I., Joseph, D., Biggley, W.H. and Seliger, H.H. (1985). Induced chemiluminescence of oxidized fatty acids and oils. Lipids, 20, 729-734.

Olcott, H.S. and Einest, E. (1958). A weighing method for measuring the induction period of marine and other oils. J. Am. Oil Chem. Soc., 35, 161-162.

Parr, L.J. and Swoboda, P.A.T. (1976). The assay of conjugable oxidation products applied to lipid deterioration in stored foods. $J$. Food Technol., 11, 1-12.

Pokorny, J., Valentova, H. and Davidek, J. (1985). Modified determination of 2-thiobarbituric acid value in fats and oils. Nahrung, 29, 31-38

Porter, N.A., Lehman, L.S., Weber, B.A. and Smith, K.J. (1981). Unified mechanism for polyunsaturated fatty acid autoxidation Competition of peroxy radical hydrogen atom abstraction, $\beta$ scission and cyclization. J. Am. Oil. Chem. Soc., 103, 6447-6452.

Robles-Martinez, C. Cervantes, E. and Ke, P.J. (1982). Recommended method for testing the objective rancidity development in fish based on TBARS formation. Can. Tech. Report Fish Aquatic Sci. No. 1089

Rossell, J.B. (1983). Measurement of rancidity. In "Rancidity in Foods," ed. by J.C. Allen and J. Hamilton. Applied Science. Publishers, London, pp. 21-45.

Saito, H. and Udagawa, M. (1992). Application of nuclear magnetic resonance to evaluate the oxidative deterioration of brown fish meal. J. Sci. Food Agric., 58, 135-137.

Schmedes, A. and Holmer, G. (1989). A new thiobarbituric acid (TBA) method for determining free malondialdehyde and hydroperoxides selectively as a measure of lipid peroxidation. J. Am. Oil Chem. Soc., 66, 813-817.

Schnaich, K.M. and Borgi, D.C. (1980). EPR studies in autoxidation. In "Autoxidation in Food and Biological Systems," ed. M.G. Simic and M. Karel. Plenum Press, NY, pp. 45-70

Selke, E., Moser, H.A. and Rohwedder, W.K. (1970). Tandem gas chromatography-mass spectrometry analysis of volatiles from soybean oil. J. Am. Oil Chem. Soc., 47, 393-397.

Shahidi, F. (1992). Current and novel methods for stability testing of canola oil. Inform, 3, 543

Shahidi, F. and Pegg, R.B. (1994). Hexanal as an indicator of meat flavor deterioration. J. Food Lipids, 1, 177-186.

Shahidi, F., Rubin, L.J., Diosady, L.L. and Wood, D.F. (1985). Effect of sulfanilamide on the TBA values of cured meats. J. Food Sci., 50, 274-275.

Shahidi, F., Rubin, L.J. and D'Souza, L.A. (1986). Meat flavor volatiles: A review of the composition, techniques of analysis, and sensory evaluation. CRC Crit. Rev. Food. Sci. Nutr., 24, 141-243.

Shahidi, F., Rubin, L.J. and Wood, D.F. (1987). Control of lipid oxidation in cooked ground pork with antioxidants and dinitrosyl ferrohemochrome. J. Food Sci., 52, 564-567.

Shahidi, F., Pegg, R.B. and Harris, R.(1991). Effects of nitrite and sulfanilamide on the 2-thiobarbituric acid (TBA) values in aqueous model and cured meat systems. J. Muscle Foods, 2, 1-9.

Shahidi, F., Wanasundara, U.N. and Brunet, N. (1994). Oxidative 
stability of oil from blubber of harp seal (Phoca groenlandica) as assessed by NMR and standard procedures. Food Res. Int., 27, 555 562.

Sherwin, E.R. (1978). Oxidation and antioxidants in fat and oil processing. J. Am. Oil Chem. Soc., 55, 809-814.

Simic, M.G. (1980). Kinetic and mechanistic studies of peroxy, vitamin $\mathrm{E}$ and antioxidant free radicals by pulse radiolysis. In "Autoxidation in Food and Biological Systems," ed. by M.G. Simic and M. Karel. Plenum Press, NY, pp. 17-26.

Snyder, J.M., Frankel, E.N. and Selke, E. (1985). Capillary gas chromatographic analyses of headspace volatiles from vegetable oils. J. Am. Oil Chem. Soc., 62, 1675-1679.

St. Angelo, A.J., Ory, R.L. and Brown, L.E. (1972). A comparison minor constituents in peanut butter as possible source of fatty acid peroxidation. Am. Peanut Res. Edu. Assoc. J., 4, 186-196.

St. Angelo, A.J., Ory, R.L. and Brown, L.E. (1975). Comparison of methods for determining peroxidation in processed whole peanut products. J. Am. Oil Chem. Soc., 52, 34-35.

St. Angelo, A.J., Vercellotti, J.R., Legendre, M.G., Vinnett, C.H., Kuan, J.W., Janies, C. Jr. and Dupuy, H.P. (1987). Chemical and instrumental analyses of warmed-over flavor in beef. J. Food. Sci. 52, 1163-1168.

Tarladgis, B.G., Pearson, A.M. and Dugan, L.R. Jr. (1964). Chemistry of the 2-thiobarbituric acid test for determination of oxidative rancidity in foods. II. Formation of the TBA-molonaldehyde complex without acid-heat treatment. J. Sci. Food Agric., 15, 602 607

Tomas, M.C. and Funes, J. (1987). Application of 2-thiobarbituric acid reaction to exudates of frozen and refrigerated meats. $J$. Food Sci., 52, 575-579.

van de Voort, F.R., Ismail, A.A., Sedman, J. and Emo, G.(1994). Monitoring the oxidation of edible oils by Fourier transform infrared spectroscopy. J. Am. Oil Chem. Soc., 71, 243-253.

Wanasundara, U.N. and Shahidi, F. (1993). Application of NMR spectroscopy to assess oxidative stability of canola and soybean oils. J. Food Lipids, 1, 15-24.

Wanasundara, U.N. and Shahidi, F. (1994). Canola extracts as an alternative natural antioxidant for canola oil. J. Am. Oil Chem. Soc., 71, 817-822.

Wanasundara, U.N. and Shahidi, F. (1995a). Storage stability of microencapsulated seal blubber oil. J. Food Lipids, 2, 73-86.

Wanasundara, U.N. and Shahidi, F. (1995b). Stability of edible oils as reflected in their propanal and hexanal contents. Presented at the Canadian Section of American Oil Chemists' Society Annual Meeting, Guelph, ON, November 15-16.

Wanasundara, U.N., Shahidi, F. and Jablonski, C.R. (1995). Comparison of standard and NMR methodologies for assessment of oxidative stability of canola and soybean oils. Food Chem., 52, 249253.

Warner, K., Evans, C.D., List, G.R., Boundy, B.K. and Kwolek, W.F. (1974). Pentane formation and rancidity in vegetable oils and potato chips. J. Food Sci., 39, 761-765.

Yukawa, N., Takamura, H. and Matoba, T. (1993). Determination of total carbonyl compounds in aqueous media. J. Am. Oil Chem. Soc., 70, 881-884 